\title{
LOCAL SYSTEMS IN GROUPS
}

\section{Dedicated to the memory of Hanna Neumann}

\author{
J. A. HULSE
}

(Received 27 June 1972)

Communicated by M. F. Newman

\section{Introduction}

Throughout this paper we will assume that all groups are contained in some fixed but arbitrary universe. Thus the class $\mathfrak{D}$ of all groups becomes a set. If $\mathfrak{X}$ is a class of groups then we assume that $1 \in \mathfrak{X}$ and if $H \cong G \in \mathfrak{X}$ then $H \in \mathfrak{X}$. German capitals will be used to denote classes of groups.

We will use an extended form of the notation for closure operators on the set of all classes of groups introduced by Hall in [1]. If $H$ is any set, let $\mathscr{P}(H)$ be the set of all subsets of $H$.

Definition 1.1. If $\mathscr{P} \subseteq \mathscr{P}(H)$ for some set $H$, a map $A: \mathscr{P} \rightarrow \mathscr{P}$ is called an operator on $\mathscr{P}$ if $X \subseteq A(X) \subseteq A(Y)$ for all $X, Y \in \mathscr{P}$ with $X \subseteq Y$. If also $\alpha$ is an ordinal number the operators $A^{\alpha}$ and $A^{*}$ on $\mathscr{P}$ are defined by $A^{0}(X)=X$, $A^{\alpha}(X)=A\left(\cup\left\{A^{\beta}(X) \mid \beta<\alpha\right\}\right)$ if $\alpha>0$ and $A^{*}(X)=\cup\left\{A^{\alpha}(X) \mid \alpha\right.$ is an ordinal $\}$ for all $X \in \mathscr{P} . A$ is called a closure operator if $A=A^{*}$.

A relation of partial order may be defined on the set of all such operators on $\mathscr{P}$ by the rule that $A \leqq B$ if and only of $A(X) \subseteq B(X)$ for all $X \in \mathscr{P}$. Then clearly $A^{\alpha} \leqq A^{\alpha+1} \leqq A^{*}$ for all ordinals $\alpha$ and if, for some $\alpha, A^{\alpha}=A^{\alpha+1}$ then $A^{\alpha}=A^{\beta}=A^{*}$ for all $\beta \geqq \alpha$. From this we see that an operator on $\mathscr{P}$ is a closure operator if and only if $A=A^{2}$. Further since $\mathscr{P}$ is a set it is clear that there exists an ordinal $\alpha$ such that $A^{\alpha}=A^{\alpha+1}=A^{*}$. Thus it follows that $A^{*}$ is a closure operator and is the least closure operator $B$ such that $A \leqq B$.

We will use small capitals to denote operators on the set of all classes of groups (in a given universe) and Roman capitals to denote operators on $\mathscr{P}(\mathscr{P}(K)$ ) for some set $K$.

In this paper we are concerned with operators derived from various types of local systems. 
Definition 1.2. A set 2 of subsets of a set $H$ is called a local system for $H$ if $\varnothing \neq \mathscr{Q}$ and each finite subset of $H$ is contained in some member of 2.2 is called weakly upper-directed if for each $Q \in \mathscr{Q}$ and each $h \in H$ there exists $R \in \mathscr{Q}$ such that $Q \cup\{h\} \subseteq R$ and $\mathscr{Q}$ is called a tower if $\mathscr{Q}$ is well-ordered by inclusion.

Definition 1.3. The operators $L_{,}, L_{w}, L_{u}, L_{t}$ are defined by $G \in L \mathfrak{X}\left(L_{w} \mathfrak{X}\right.$, $L_{\mathbf{u}} \mathfrak{X}, L_{\mathrm{t}} \mathfrak{X}$, respectively) if and only if $G$ has a local system $\mathscr{Q}$ of $\mathfrak{X}$-subgroups (which is weakly upper-directed, upper-directed, a tower, respectively) for all classes of groups $\mathfrak{X}$. The operators $\mathrm{L}, \mathrm{L}_{\mathbf{w}}, \mathrm{L}_{\mathbf{u}}, \mathrm{L}_{\mathbf{t}}$ on $\mathscr{P}(\mathscr{P}(K))$ are defined similarly with $\mathscr{P}(\mathscr{P}(K))$ replacing the set of all classes of groups.

We remark that in our definition of a local system we are following Hartley [2]. In [4] Kuroš requires every local system to be upper-directed.

It follows immediately from the definition, that

$$
\mathrm{L}_{\mathrm{t}} \leqq \mathrm{L}_{\mathrm{u}} \leqq \mathrm{L}_{\mathbf{w}} \leqq \mathrm{L}
$$

and hence for all ordinals $\alpha$

$$
L_{\mathrm{t}}^{\alpha} \leqq \mathrm{L}_{\mathrm{u}}^{\alpha} \leqq \mathrm{L}_{\mathrm{w}}^{\alpha} \leqq \mathrm{L}^{\alpha} \text { and } \mathrm{L}_{\mathrm{t}}^{*} \leqq \mathrm{~L}_{\mathrm{u}}^{*} \leqq \mathrm{~L}_{\mathrm{w}}^{*} \leqq \mathrm{~L}^{*} .
$$

It is well known that $\mathrm{L}=\mathrm{L}^{*}$ and $\mathrm{L}_{\mathrm{u}} \mathfrak{X}=\mathrm{L}_{\mathrm{u}}^{*} \mathfrak{X}$ if $\mathfrak{X}=\mathrm{s} \mathfrak{X}$, where $G \in \mathrm{S} \mathfrak{X}$ if and only if $G$ is a subgroup of some $\mathfrak{X}$-group. In [3] Hickin showed that $L_{\mathbf{u}}<\mathbf{L}_{\mathbf{u}}^{*}$. The first aim of this paper is to show that the inequalities in (1) and (2) are all strict, with the exception that $L_{\mathfrak{i}}^{*}=L_{u}^{*}$ and also that $A^{\alpha}<A^{\alpha+1}<A^{*}$ in the cases where $A=L_{t}, L_{u}$ and $L_{w}$ with some restriction on the cardinality, $|\alpha|$, of the ordinals $\alpha$. These results are given in

Theorem A. Let $\alpha$ be an ordinal number. Then:

(i) $\mathbf{L}_{w}^{*}<\mathbf{L}$;

(ii) if $|\alpha| \leqq \aleph_{0}$ then $\mathrm{L}_{\mathrm{w}}^{\alpha}<\mathrm{L}_{\mathrm{w}}^{\alpha+1}<\mathrm{L}_{\mathrm{w}}^{*}$;

(iii) $\mathrm{L}_{\mathrm{u}}^{*}<\mathrm{L}_{\mathrm{w}}^{*}$ and $\mathrm{L}_{\mathrm{u}}^{\alpha}<\mathrm{L}_{\mathrm{w}}^{\alpha}$;

(iv) (Hickin) if $|\alpha| \leqq 2^{N_{0}}$ then $\mathrm{L}_{\mathrm{u}}^{\alpha}<\mathrm{L}_{\mathrm{u}}^{\alpha+1}<\mathrm{L}_{\mathrm{u}}^{*}$;

(v) $\mathrm{L}_{\mathrm{u}} \mathrm{L}_{\mathrm{i}}^{\alpha}, \mathrm{L}_{\mathrm{i}}^{\alpha}<\mathrm{L}_{\mathrm{u}}^{\alpha}$ and $\mathrm{L}_{\mathrm{i}}^{\alpha}<\mathrm{L}_{\mathrm{t}}^{\alpha+1}<\mathrm{L}_{\mathrm{t}}^{*}$;

(vi) $\mathbf{L}_{\mathbf{i}}^{*}=\mathbf{L}_{\mathbf{u}}^{*}$.

Thus, in particular, the operators $L_{t}, L_{u}$ and $L_{w}$ are not closure operators and $\mathrm{L}_{\mathrm{t}}^{*}=\mathrm{L}_{\mathrm{u}}^{*}<\mathrm{L}_{\mathrm{w}}^{*}<\mathrm{L}^{*}=\mathrm{L}$. In Section 2 of the paper we prove some purely set theoretic lemmas required for the proof of Theorem A while in Section 3 we develop the necessary translation into group theory.

This investigation was sparked off by the question as to whether the class $\mathfrak{L}_{s}$, the class of all groups $G$ in which the set of all serial subgroups of $G$ forms a complete sublattice of the lattice of all subgroups of $G$, is L-closed. This question arose from the work of Hartley in [2] where he showed that $\mathfrak{L}_{\mathrm{s}}$ contains all 
locally finite groups. We have been unable to completely decide this question but we will prove the following results in Section 4.

THEOREM B. The class $\mathfrak{L}_{\mathrm{s}}$ is $\mathrm{L}_{\mathrm{w}}{ }^{*}$-closed and if $\mathfrak{X}=\mathrm{s} \mathfrak{X} \subseteq \mathfrak{L}_{\mathrm{s}}$ then $\mathrm{L} \mathfrak{X} \subseteq \mathfrak{L}_{\mathrm{s}}$.

We remark that no such local theorem can hold for the classes $\mathfrak{L}_{n}, \mathfrak{L}_{\mathrm{d}}$ or $\mathfrak{L}_{\mathrm{a}}$, consisting of all groups in which the set of all subnormal, ascendant or decendant subgroups, respectively, forms a complete sublattice of the lattice of all subgroups, since there exist locally nilpotent groups in which the join of two subnormal subgroups is neither ascendant nor descendant - see for example [7, Appendix D, Exercise 23].

If $\mathfrak{M}^{\vee}$ and $\mathfrak{M}_{\mathrm{s}}^{\vee}$ are the classes of groups with the minimum condition on all subgroups and all serial subgroups, respectively, then we will deduce the following extension of Theorem A of [2] as a corollary of Theorem B.

THEOREM C. $\mathfrak{M}_{\mathrm{s}}^{\vee} \subseteq \mathfrak{L}_{\mathrm{s}}$ and $\mathrm{L} \mathfrak{M}^{\vee} \subseteq \mathfrak{L}_{\mathrm{s}}$.

It is perhaps worth noting in contrast that an example in [2] shows that there are groups with the maximum condition on all subgroups which do not lie in $\mathfrak{R}_{\mathrm{s}}$.

\section{The set theory}

We begin this section with

LEMMA 2.1. For each ordinal $\alpha$ and each set $K$ with $|K| \geqq \max \left\{\aleph_{0},|\alpha|\right\}$ there exists a subset $\mathscr{2}$ of $\mathscr{P}(K)$ such that

(i) $\varnothing \in \mathscr{Q}$;

(ii) $\mathbf{L}_{\mathbf{u}}{ }^{\beta}(\mathscr{Q})=\mathbf{L}_{\mathrm{w}}{ }^{\beta}(\mathscr{Q})$ for all $\beta$;

(iii) $\mathrm{L}_{\mathrm{u}}{ }^{\beta}(\mathscr{Q}) \subset \mathrm{L}_{\mathrm{u}}{ }^{\alpha}(\mathscr{Q})=\mathrm{L}_{\mathrm{u}}{ }^{*}(\mathscr{Q})$ if $\beta<\alpha$.

Proof. (iii) is just the contents of Lemma 1 of [3], (ii) is easily seen to follow from the construction in [3] and (i) follows since $\mathrm{L}_{\mathrm{u}}(\mathscr{Q} \cup\{\varnothing\})=\mathrm{L}_{\mathrm{u}}(\mathscr{Q}) \cup\{\varnothing\}$ and $\mathbf{L}_{\mathbf{w}}(\mathscr{Q} \cup\{\varnothing\})=\mathbf{L}_{\mathbf{w}}(\mathscr{Q}) \cup\{\varnothing\}$.

LEMMA 2.2. If $K$ is any set with $|K| \geqq \aleph_{0}$ then there exists a subset $\mathscr{2}$ of $\mathscr{P}(K)$ such that $\varnothing \in \mathscr{Q}$ and

$$
\mathbf{L}_{\mathbf{w}}^{*}(\mathscr{Q}) \subset \mathbf{L}(\mathscr{Q}) .
$$

Proof. Let $\mathscr{F}$ be the set of all finite subsets of $K$. Then $|\mathscr{F}|=|K|$ since $K$ is infinite. We now partition $K$ into sets $K_{F}$, one for each $F \in \mathscr{F}$, with $\left|K_{F}\right|=|K|$ and let

$$
\mathscr{Q}=\{\varnothing\} \cup\left\{F \cup K_{F} \mid F \in \mathscr{F}\right\},
$$

so that $\varnothing \in \mathscr{Q} \subseteq \mathscr{P}(K)$. Suppose now that $Q, R \in \mathscr{Q} \backslash\{\varnothing\}$ and $Q \subseteq R$. Then $Q=E \cup K_{E}$ and $R=F \cup K_{F}$ for some $E, F \in \mathscr{F}$, so $K_{E} \subseteq F \cup K_{F}$ and so, 
since $|F|<\aleph_{0} \leqq\left|K_{E}\right|, K_{E} \cap K_{F} \neq \varnothing$. Thus $E=F$ and so $Q=R$. Hence it follows that any weakly upper-directed local system which is contained in 2 must have at most one member distinct from $\varnothing$, so $\mathrm{L}_{\mathrm{w}}(2)=\mathscr{2}$ and so $\mathrm{L}_{\mathrm{w}}{ }^{*}(\mathscr{Q})=\mathscr{Q}$. However, $\mathscr{Q}$ is a local system for $K$ and $K \notin \mathscr{Q}$ and so $\mathscr{Q} \subset \mathrm{L}(\mathscr{Q})$, as required.

LEMMA 2.3. If $K$ is any set with $|K|=\aleph_{\alpha}$ for some non-limit ordinal $\alpha \geqq 1$ then there exists a set $\mathscr{Q} \subseteq \mathscr{P}(K)$ such that $\varnothing \in \mathscr{Q}$ and

$$
\mathscr{Q}=\mathrm{L}_{\mathbf{u}}{ }^{*}(\mathscr{Q}) \subset \mathrm{L}_{\mathrm{w}}(\mathscr{Q}) .
$$

Proof. We are indebted to the referee for the following proof which is much shorter than our original. By Theorem 1 on p. 451 of Sierpinski [7] there exists a set $\mathscr{R} \subseteq \mathscr{P}(K)$ such that

$$
|\mathscr{R}|>\aleph_{\alpha} \text { and }|R|=\aleph_{\alpha} \text { for all } R \in \mathscr{R}
$$

and

$$
\text { if } S, T \in \mathscr{R} \text { and } S \neq T \text { then }|S \cap T|<\aleph_{\alpha} .
$$

Now clearly $\mathscr{R}$ also satisfies

(3) if $S, T_{1}, T_{2}, \cdots$ are countably many distinct elements of $\mathscr{R}$ then $S \notin \bigcup_{n=1}^{\infty} T_{n}$.

For each $x \in K$ let $\mathscr{R}_{x}=\{R \in \mathscr{R} \mid x \in R\}$ and let $\bar{K}=\left\{x \in K|| \mathscr{R}_{x} \mid<\aleph_{\alpha}\right\}$. Then $\left|\cup\left\{\mathscr{R}_{x} \mid x \in R\right\}\right| \leqq \aleph_{\alpha}$ and so by (1) we may replace $K$ by $K \backslash R$ and $\mathscr{R}$ by $\mathscr{R} \backslash \cup\left\{\mathscr{R}_{x} \mid x \in R\right\}$ in order to assume

$$
\left|\mathscr{R}_{x}\right| \geqq \aleph_{\alpha} \text { for all } x \in K \text {. }
$$

Let

$$
M=\{(x, n) \mid x \in K, n=0,1,2, \cdots\} .
$$

Since $|M|=|K|=\aleph_{\alpha}$ we may well-order $M=\left\{t_{\beta} \mid \beta<\omega_{\alpha}\right\}$. Now by using (4) we may choose, by a transfinite construction, distinct $R_{\beta} \in \mathscr{R}$ for each $\beta<\omega_{\alpha}$ such that $x \in R_{\beta}$ where $t_{\beta}=(x, n)$. We shall write $R_{\beta}=R_{x}^{n}$ if $t_{\beta}=(x, n)$.

We now define the sets $\mathscr{Q}_{n}$ for $n \leqq \omega$ by

and take

$$
\mathscr{Q}_{n}=\left\{\bigcup_{i<n} \dot{R}_{x_{i}}^{i} \mid x_{\imath} \in K\right\}
$$

$$
\mathscr{Q}=\bigcup_{n \leqq \infty} \mathscr{Q}_{n}
$$

Thus $\varnothing \in \mathscr{Q}_{0} \subseteq \mathscr{Q} \subseteq \mathscr{R}$ and $\bigcup_{n<\omega} \mathscr{Q}_{n}$ is a weakly upper-directed local system for $K$, giving $K \in \mathrm{L}_{\mathrm{w}}(\mathscr{2})$. But by (2) $K \notin \mathscr{R}$ and so $\mathscr{Q} \subset \mathrm{L}_{\mathrm{w}}(\mathscr{Q})$.

It now remains to show that $\mathscr{Q}=\mathrm{L}_{\mathrm{u}}{ }^{*}(\mathscr{Q})$. Let $Q_{1}, Q_{2} \in \mathscr{Q}$ and $Q_{1} \subseteq Q_{2}$. Then there exist $m, n$ with $0 \leqq m, n \leqq \omega$ and $x_{i}, y_{j} \in K$ for $0 \leqq i<m$ and $0 \leqq j<n$ 
such that

$$
Q_{1}=\bigcup_{i<m} R_{x_{i}}^{i} \text { and } Q_{2}=\bigcup_{j<n} R_{y_{j}}^{j} .
$$

Then by (3) we must have $m \leqq n$ and $x_{i}=y_{i}$ for $0 \leqq i<m$. Hence it follows that $\mathrm{L}_{\mathrm{u}}(\mathscr{Q})=\mathscr{Q}$ and so $\mathrm{L}_{\mathrm{u}}{ }^{*}(\mathscr{Q})=\mathscr{Q}$, as required.

We remark that it is easy to see that if $|K| \leqq \aleph_{0}$ and $\mathscr{Q} \subseteq \mathscr{P}(K)$ then $\mathrm{L}_{\mathrm{t}}(\mathscr{Q})=\mathrm{L}_{\mathbf{u}}(\mathscr{Q})=\mathrm{L}_{\mathbf{w}}(\mathscr{Q})$ and so we certainly require $|K| \geqq \aleph_{1}$ for Lemma 2.3 to hold.

\section{The group theory}

We begin with two lemmas, the first of which is proved in [3] except for the part concerning the operator $\mathrm{L}_{\mathbf{w}}$ which follows easily.

LeMMA 3.1. Let $K$ be any set with $|K|=2^{N_{0}}$. Then there exist groups $F_{Q}$ for each $Q \subseteq K$, where $F_{\varnothing}=1$, such that the map

$$
\phi: \mathscr{Q} \mapsto \mathfrak{Q}=\left\{G \mid G \cong F_{\mathbb{Q}} \text { for some } Q \in \mathscr{Q}\right\}
$$

is a bijection from the set of all $\mathscr{Q} \subseteq \mathscr{P}=\mathscr{P}(K)$ with $\varnothing \in \mathscr{Q}$ onto the set of all classes of groups contained in $\mathfrak{P}$. Further

$$
\phi: \mathrm{L}_{\mathbf{u}}{ }^{\alpha}(\mathscr{Q}) \mapsto \mathrm{L}_{\mathbf{u}}{ }^{\alpha}(\mathscr{Q}) \cap \mathfrak{P},
$$

for all ordinals $\alpha$ and all $\mathscr{Z}$ with $\varnothing \in \mathscr{Q} \subseteq \mathscr{P}$, and $\phi$ maps $\mathrm{L}_{\mathrm{w}}(\mathscr{Q})$ onto a subset of $\mathbf{L}_{\mathbf{w}}(\mathfrak{Q})$.

Since, if $Q \neq \varnothing$, the groups $F_{Q}$ are not finitely generated the argument used by Hickin will not work in full for the operators $L_{w}$ and $L$. Also, since it is easy to see that $L_{1}(\mathfrak{P}) \supset \mathfrak{P}$, the method of [3] will not decide precisely where the sequence $\mathrm{L}_{\mathrm{u}}^{\alpha}(\mathbb{Q})$ terminates but only give a lower bound. For these reasons we establish the following lemma which avoids these difficulties at the expense of a further restriction on the cardinality of $K$.

LemMA 3.2. Let $K$ be a set with $|K|=\aleph_{0}$. Then there exist groups $A_{Q}$ for each $Q \subseteq K$, with $A_{\varnothing}=1$, such that the map

$$
\phi: \mathscr{Q} \mapsto \mathscr{Q}=\left\{G \mid G \cong A_{\mathcal{Q}} \text { for some } Q \in \mathscr{Q}\right\}
$$

is a bijection from the set of all $\mathscr{Q} \subseteq \mathscr{P}=\mathscr{P}(K)$ with $\varnothing \in \mathscr{Q}$ onto the set of all classes of groups contained in $\mathfrak{P}$. Further

$$
\begin{gathered}
\phi: \mathrm{L}_{\mathrm{t}}^{\alpha}(\mathscr{Q}) \mapsto \mathrm{L}_{\mathrm{t}}^{\alpha}(\mathbb{Q}), \phi: \mathrm{L}_{\mathrm{u}}^{\alpha}(\mathscr{Q}) \mapsto \mathrm{L}_{\mathrm{u}}^{\alpha}(\mathbb{Q}), \\
\phi: \mathrm{L}_{\mathrm{w}}^{\alpha}(\mathscr{Q}) \mapsto \mathrm{L}_{\mathrm{w}}^{\alpha}(\mathbb{Q}) \text { and } \phi: \mathrm{L}(\mathscr{Q}) \mapsto \mathrm{L}(\mathbb{Q}) \subseteq \mathfrak{P}
\end{gathered}
$$

for all ordinals $\alpha$ and all $\mathscr{Q}$ with $\varnothing \in \mathscr{Q} \subseteq \mathscr{P}$. 
Proof. Since $|K|=\aleph_{0}$ we may assume that $K$ is the set of all prime numbers. For each $k \in K$ let $C_{k}$ be a cyclic group of order $k$. If $Q \subseteq K$ let $A_{Q}$ be the restricted direct product of the groups $C_{k}$ with $k \in Q$, so that $A_{\varnothing}=1$.

We first show that $\mathfrak{P}=\mathbf{L P}$. Since $\mathfrak{B}$ is contained in the class of locally finite Abelian groups so is $\mathrm{L} \mathfrak{P}$. But if $G \in \mathrm{L} \mathfrak{P}$ and has a Sylow $p$-subgroup of order greater than $p$ then $G$ has a subgroup $H$ of order $p^{2}$ and so there exists a subgroup $B$ of $G$ containing $H$ and isomorphic with $A_{Q}$ for some $Q$. This is a contradiction as the Sylow $p$-subgroup of $B$ has order at most $p$. So if $G \in \mathrm{L} \mathfrak{B}$ then $G$ is a locally finite Abelian group with Sylow $p$-subgroups of order at most $p$ for each prime $p$. Thus $G \in \mathfrak{P}$ and $\mathfrak{P}=L \mathfrak{P}$.

We next show that if $C \cong A_{Q}$ and $C \leqq A_{R}$ then $Q \subseteq R$ and $C=A_{Q}$. For if $p \in Q$ then the Sylow $p$-subgroup of $C$ has order $p$. But the Sylow $p$-subgroup of $A_{R}$ has order $p$ or 1 depending on whether $p \in R$ or $p \notin R$. Now since $C \leqq A_{R}$ it follows that $Q \subseteq R$ and $C=A_{Q} \leqq A_{R}$.

Hence if $\mathscr{Q}$ is a set of $\mathfrak{P}$-subgroups of $A_{R}$ then $\mathscr{Q} \subseteq\left\{A_{Q} \mid Q \subseteq R\right\}$. It is now easy to deduce that for each $R \subseteq K$ the map

$$
\mathscr{Q} \mapsto\left\{A_{Q} \mid Q \in \mathscr{Q}\right\}
$$

is a bijection from the set of all local systems $\mathscr{Q}$ for $R$ with $\mathscr{Q} \subseteq \mathscr{P}$ onto the set of all local systems of $\mathfrak{P}$-subgroups for $A_{R}$ which preserves the properties of being weakly upper-directed, upper-directed and a tower. Thus the lemma holds for $\alpha=1$ and the rest follows by induction.

The corollary below follows immediately from Lemmas 2.1 and 3.2.

Corollary 3.3. Let $\alpha$ be an ordinal with $|\alpha| \leqq \aleph_{0}$. Then there exists a class of groups $\mathbb{Q}_{\alpha}$ such that $\mathrm{L}_{\mathrm{u}}{ }^{\beta}\left(\mathfrak{Q}_{\alpha}\right)=\mathrm{L}_{\mathrm{w}}{ }^{\beta}\left(\mathfrak{Q}_{\alpha}\right)$ for all $\beta$ and

$$
\mathrm{L}_{\mathbf{u}}{ }^{\gamma}\left(\mathbb{Q}_{\alpha}\right) \subset \mathbf{L}_{\mathbf{u}}{ }^{\alpha}\left(\mathfrak{Q}_{\alpha}\right)=\mathbf{L}_{\mathbf{u}}{ }^{*}\left(\mathbb{Q}_{\alpha}\right)
$$

for all $\gamma<\alpha$.

Before proving Theorem A we establish one further lemma.

Lemma 3.4. If for each ordinal $\alpha, D_{\alpha}$ is the class of all groups of cardinality at most $\aleph_{\alpha}$ then $\mathrm{L}_{\mathrm{u}}\left(\mathfrak{D}_{0}\right)=\mathfrak{D}$ and $\mathrm{L}_{\mathrm{t}}{ }^{\alpha}\left(\mathfrak{D}_{0}\right)=\mathfrak{D}_{\alpha}$.

Proof. Since a subgroup of a group $G$ which is generated by a finite or countable subset is at most countable, the set of all $D_{0}$-subgroups of $G$ is an upperdirected local system for $G$ and so $\mathrm{L}_{\mathrm{u}}\left(\mathfrak{D}_{\mathfrak{0}}\right)=\mathfrak{D}$.

We prove the second part by induction on $\alpha$, the result being true by hypothesis if $\alpha=0$. Suppose now that $\alpha>0$ and $\mathrm{L}_{\mathrm{t}}^{\beta}\left(\mathfrak{D}_{0}\right)=\mathfrak{D}_{\beta}$ for all $\beta<\alpha$. Let $G \in D_{\alpha}$ then by the induction hypothesis we may assume that $|G|=\aleph_{\alpha}$ and so $G=\left\{g_{\gamma} \mid \gamma<\omega_{\alpha}\right\}$. If $\theta<\omega_{\alpha}$ let $G_{\theta}=\left\langle g_{\gamma} \mid \gamma<\theta\right\rangle$ then $\left|G_{\theta}\right|<\aleph_{\alpha}$ and so by induction $G_{\theta} \in \cup\left\{\mathrm{L}_{\mathrm{t}}{ }^{\beta}\left(\mathfrak{D}_{0}\right) \mid \beta<\alpha\right\}$. But $G_{\theta} \leqq G_{\phi}$ if $\theta<\phi<\omega_{\alpha}$ and 
$G=\cup\left\{G_{\theta} \mid \theta<\omega_{\alpha}\right\}$, so $G \in \mathrm{L}_{t}^{\alpha}\left(\mathcal{D}_{0}\right)$ and so $\mathfrak{D}_{\alpha} \subseteq \mathrm{L}_{\mathrm{t}}^{\alpha}\left(\mathfrak{D}_{0}\right)$. Conversely if $G \in \mathrm{L}_{t}^{\alpha}\left(\mathfrak{D}_{0}\right)$ then there exist an ordinal $\gamma$ and subgroups $G_{\theta} \in \cup\left\{\mathrm{L}_{\mathbf{t}}^{\beta}\left(\mathfrak{D}_{0}\right) \mid \beta<\alpha\right\}$ such that $G_{\theta}<G_{\phi}$ for all $\theta<\phi<\gamma$ and $G=\cup\left\{G_{\theta} \mid \theta<\gamma\right\}$. Thus by induction $|\theta| \leqq\left|G_{\theta}\right|<\aleph_{\alpha}$ for all $\theta<\gamma$ and so $|\gamma| \leqq \aleph_{\alpha}$.

Hence $|G| \leqq|\gamma| \max \left\{\left|G_{\theta}\right| \mid \theta<\gamma\right\} \leqq \aleph_{\alpha}^{2}=\aleph_{\alpha}$, so $G \in D_{\alpha}$ and so $L_{1}^{\alpha}\left(\mathfrak{D}_{0}\right) \subseteq \mathfrak{D}_{\alpha}$. Thus the lemma holds by induction.

We end this section with the

Proof of Theorem A. (i) $L_{w}^{*}<L$ follows immediately from Lemmas 2.2 and 3.2.

(ii) $L_{\mathrm{w}}^{\alpha}<L_{\mathrm{w}}^{\alpha+1}<L_{\mathrm{w}}^{*}$ for all $\alpha$ with $|\alpha| \leqq N_{0}$ follows from Corollary 3.3.

(iii) $L_{u}{ }^{*}<L_{w}{ }^{*}$ follows from Lemmas 2.3 and 3.1 whence it is easy to deduce that $\mathrm{L}_{\mathrm{u}}{ }^{\alpha}<\mathrm{L}_{\mathrm{w}}{ }^{\alpha}$ for all ordinals $\alpha$.

(iv) $\mathrm{L}_{\mathrm{u}}{ }^{\alpha}<\mathrm{L}_{\mathrm{u}}{ }^{\alpha+1}<\mathrm{L}_{\mathrm{u}}{ }^{*}$ for all $\alpha$ with $|\alpha| \leqq 2^{\mathrm{N}_{0}}$ follows from Lemmas 2.1 and 3.1. Corollary 3.3 would give the result if $|\alpha| \leqq \aleph_{0}$.

(v) $\mathrm{L}_{\mathrm{u}} \mathrm{L}_{\mathrm{t}}{ }^{\alpha}, \mathrm{L}_{\mathrm{t}}{ }^{\alpha}<\mathrm{L}_{\mathrm{u}}{ }^{\alpha}$ and $\mathrm{L}_{\mathrm{t}}{ }^{\alpha}<\mathrm{L}_{\mathrm{t}}{ }^{\alpha+1}<\mathrm{L}_{\mathrm{t}}{ }^{*}$ all follow from Lemma 3.4 and the existence of groups of arbitrary cardinality.

(vi) We show that if $\mathfrak{X}=L_{\mathfrak{t}}{ }^{*}(\mathfrak{X})$ then $\mathfrak{X}=\mathrm{L}_{\mathrm{u}}{ }^{*}(\mathfrak{X})$ whence $\mathrm{L}_{\mathrm{t}}{ }^{*}=\mathrm{L}_{\mathrm{u}}{ }^{*}$ since we already have $\mathrm{L}_{\mathrm{t}}{ }^{*} \leqq \mathrm{~L}_{\mathrm{u}}{ }^{*}$. Suppose now that $G \in \mathrm{L}_{\mathrm{u}}(\mathfrak{X})$ and $\mathscr{X}$ is the set of $\mathfrak{X}$-subgroups of $G$. Now since $\mathfrak{X}=\mathrm{L}_{\mathfrak{t}}{ }^{*}(\mathfrak{X}), \cup \mathscr{T} \in \mathscr{X}$ for any nonempty tower $\mathscr{T} \subseteq \mathscr{X}$. Since $G \in \mathrm{L}_{\mathbf{u}}(\mathfrak{X})$ there exists an upper-directed local system $\mathscr{Q}$ for $G$ with $\mathscr{Q} \subseteq \mathscr{X}$. Now by Zorn's Lemma $G=\cup \mathscr{Q} \in \mathscr{X}$ and so $G \in \mathfrak{X}$. Hence $L_{u}(\mathfrak{X})=\mathfrak{X}$ and so $\mathrm{L}_{\mathbf{u}}{ }^{*}(\mathfrak{X})=\mathfrak{X}$, as required.

\section{The class $\mathfrak{L}_{\mathrm{s}}$}

We begin with

Proof of Theorem B. Suppose that a group $G$ has a weakly upper-directed local system $\mathscr{Q}$ of $\mathfrak{Q}_{\mathrm{s}}$-subgroups, $\mathscr{H}$ is a set of serial subgroups of $G$ and $J=\langle\mathscr{H}\rangle$ $=\langle H \mid H \in \mathscr{H}\rangle$. We show first that $J \cap Q$ ser $Q$ for all $Q \in \mathscr{Q}$, i.e. $J \cap Q$ is a serial subgroup of $Q$.

Let $Q \in \mathscr{Q}$ and $x \in J \cap Q \leqq J$. Then there exist $H_{1}, \cdots, H_{n} \in \mathscr{H}$ and finitely generated subgroups $H_{i}{ }^{*}$ of each $H_{i}$ such that $x \in\left\langle H_{1}{ }^{*}, \cdots, H_{n}{ }^{*}\right\rangle$. Now since $\mathscr{Q}$ is weakly upper-directed there exists $R \in \mathscr{Q}$ such that

$$
\left\langle Q, H_{1}{ }^{*}, \cdots, H_{n}{ }^{*}\right\rangle \leqq R .
$$

Let

$$
K=\left\langle H_{1} \cap R, \cdots, H_{n} \cap R\right\rangle .
$$

Now $H_{i} \cap R$ ser $R$ for $1 \leqq i \leqq n$ and $R \in \mathcal{Q}_{\mathrm{s}}$, so $K$ ser $R$ and so, since $Q \leqq R$, $K \cap Q$ ser $Q$. But, since $H_{i}{ }^{*} \leqq H_{i} \cap R \leqq J, x \in K \cap Q \leqq J \cap Q$. Thus $J \cap Q$ is the join of all its subgroups which are serial in $Q \in \mathfrak{L}_{\mathrm{s}}$ and so $J \cap Q \operatorname{ser} Q$. Hence by Lemma 1 of [2], $J$ ser $G$. Thus $\mathrm{L}_{\mathrm{w}}\left(\mathfrak{L}_{\mathrm{s}}\right)=\mathfrak{L}_{\mathrm{s}}$ and so $\mathrm{L}_{\mathrm{w}}{ }^{*}\left(\mathfrak{L}_{\mathrm{s}}\right)=\mathfrak{L}_{\mathrm{s}}$. 
Finally if $\mathfrak{X}=s \mathfrak{X} \subseteq \mathfrak{L}_{\mathrm{s}}$ then $L \mathfrak{X}=L_{\mathrm{u}} \mathfrak{X}$ and so $L \mathfrak{X} \subseteq \mathfrak{L}_{\mathrm{s}}$.

We will require one further lemma for the proof of Theorem $\mathbf{C}$.

LemMA 4.1. Let $\mathscr{H}$ be a set of finite ascendant subgroups of a group $G$. Then $\langle\mathscr{H}\rangle \operatorname{ser} G$.

Proor. If $J=\langle\mathscr{H}\rangle$ and $K=\left\langle J^{G}\right\rangle$ then $K$ is generated by finite ascendant subgroups of $G$ and so $K$ is locally tinite. Thus by Theorem B or Theorem A of [2], $K \in \mathfrak{L}_{\text {s }}$ and so $J$ ser $K$. But $K$ is normal in $G$ and so $J$ ser $G$, as required.

Proof of Theorem C. Suppose that $G \in \mathfrak{M}_{\mathrm{s}}^{\mathrm{v}}, \mathscr{H}$ is a set of serial subgroups of $G$ and $J=\langle\mathscr{H}\rangle$. Then each $H \in \mathscr{H}$ is necessarily an ascendant subgroup of $G$ and there exists a normal subgroup $H^{*}$ of finite index in $H$ and having no finite quotient groups. Then by Lemma 4.3 of [5] and its Corollary (cf. Lemma 4 of [6]), $J^{*} \triangleleft\left\langle J^{* G}\right\rangle \triangleleft G$ where $J^{*}=\left\langle H^{*} \mid H \in \mathscr{H}\right\rangle$.

Suppose now that $L=\left\langle J^{* G}\right\rangle$ and $M=\left\langle J^{* J}\right\rangle$. Then $M \triangleleft J L, M \leqq J$ and $J / M$ is generated by finite ascendant subgroups of $J L / M$ and so by Lemma 4.1, $J$ ser $J L$. Further $J L / L$ is generated by finite ascendant subgroups of $G / L$ and so by Lemma $4.1, J L$ ser $G$. Hence $J$ ser $G$ and so $\mathfrak{M}_{\mathrm{s}}^{v} \subseteq \mathfrak{L}_{\mathrm{s}}$.

Finally since $\mathfrak{M}^{\vee}=s \mathfrak{M}^{\vee}$, Theorem $B$ shows that $L \mathfrak{M}^{\vee} \subseteq \mathfrak{L}_{\mathrm{s}}$.

\section{References}

[1] P. Hall, 'On non-strictly simple groups', Proc. Cambridge Philos. Soc. 59 (1963), 531-553.

[2] B. Hartley, 'Serial subgroups of locally finite groups', Proc. Cambridge Philos. Soc. 71 (1972), 199-201.

[3] K. K. Hickin, 'A class of groups whose local sequence is nonstationary', Proc. Amer, Math. Soc. 21 (1969), 402-408.

[4] A. G. Kuros, The Theory of Groups, Vol. 2 (Chelsea, New York, 1956).

[5] D. J. S. Robinson, 'On the theory of subnormal subgroups', Math. Zeit. 89 (1965), 30-51.

[6] J. E. Roseblade, 'On certain subnormal coalition classes', J. Algebra 1 (1964), 132-138.

[7] W. Sierpinski, Cardinal and Ordinal Numbers (Monografie Matematyczne, Tom 34, Polish Scientific Publishers, Warsaw, 2nd Ed. Revised 1965).

[8] H. Zassenhaus, The Theory of Groups, (Chelsea, New York, 2nd Ed. 1958).

Mathematical Institute

University of Edinburgh

Scotland. 